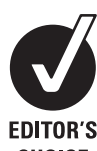

CHOICE

See Editorial, page 663

- Additional Methods and Results data are published online only at http://thorax.bmj. com/content/vol63/issue8

${ }^{1}$ Division of Infection and Immunity, School of Clinical

Science, University of Liverpool, Liverpool, UK; ${ }^{2}$ Clinical Science

Centre, University Hospital

Aintree, Liverpool, UK

Correspondence to:

Dr P P Walker, Clinical Science

Centre, University Hospital

Aintree, Lower Lane, Liverpool

L9 7AL, UK; ppwalker@liv.ac.uk

Received 11 July 2007

Accepted 25 March 2008

Published Online First

16 May 2008

\title{
Lower limb activity and its determinants in COPD
}

\author{
P P Walker, ${ }^{1,2}$ A Burnett, ${ }^{1}$ P W Flavahan, ${ }^{1}$ P M A Calverley ${ }^{1}$
}

\begin{abstract}
Background: Patients with chronic obstructive pulmonary disease (COPD) walk less than healthy older people and their self-reported activity predicts exacerbation risk. The relationship between lower limb activity and total daily activity is not known, nor are there any data which relate objectively assessed daily activity to laboratory assessments made before and after rehabilitation.
\end{abstract}

Methods: Lower limb activity was measured by leg actigraphy over 3 days in 45 patients with moderate to severe COPD and 18 controls of similar age. Thirty-three patients with COPD entered an 8-week rehabilitation programme in which the change in leg activity was measured and related to other outcomes.

Results: In patients with COPD the mean level of activity measured by whole body and leg activity monitors was closely related $(r=0.92 ; p<0.001)$, but leg activity was consistently reduced compared with controls of similar age $(p=0.001)$. Mean leg activity, mean intensity of leg activity and the time that patients spent mobile at home were all related to forced expiratory volume in $1 \mathrm{~s}\left(\mathrm{FEV}_{1}\right)$ $(r=0.57, p=0.001 ; r=0.5, p=0.003$; and $r=0.51$, $p=0.002$, respectively), but intensity of activity and time spent mobile were not related. Subjects completing pulmonary rehabilitation showed significant improvements in mean activity $(p=0.001)$ and spent more time moving $(p=0.014)$. These changes were unrelated to improvement in muscle strength or walking distance but correlated with baseline FEV $(r=0.8, p<0.001)$.

Conclusions: Total daily activity in patients with COPD is closely related to leg activity which is reduced compared with controls of similar age. Individuals differ in the time spent mobile during the day, but subjective and objectively assessed activity improves after rehabilitation and is predicted by FEV 1 . The change in activity is unrelated to improvements in corridor walking and health status.

Symptomatic chronic obstructive pulmonary disease (COPD) is associated with impaired exercise performance which, in turn, is related to a reduction in health status ${ }^{1}$ and mortality. ${ }^{2}$ Conventionally, this impairment has been documented by incremental or endurance exercise testing often using field exercise tests such as the 6 min walking distance ${ }^{3}$ or the endurance shuttle walking test. ${ }^{4}$ However, exercise testing measures what an individual is capable of doing rather than their activity. The level of activity reported by patients with COPD relates to the risk of hospitalisation after an exacerbation ${ }^{5}$ and mortality. ${ }^{6}$ More recently, the availability of reliable accelerometers has made it possible objectively to monitor daily activities outside the laboratory. Patients with COPD are less active than healthy age-matched controls and spend longer sitting and lying down, ${ }^{7}$ while activity improves after a rehabilitation programme irrespective of the exercise regime used. ${ }^{8}$

To date, home activity monitoring has reported total body movements over a $12 \mathrm{~h}$ period using a waist-mounted triaxial accelerometer which, in the case of the Dynaport system, also reports the type of activity. ${ }^{9}$ The amount of total daily activity resulting from lower limb movement has not been determined. This is important as patients with COPD are subject to loss of skeletal muscle mass which is most evident in the legs, ${ }^{10}$ and reduced quadriceps strength predicts both healthcare use ${ }^{11}$ and future mortality. ${ }^{12}$

We hypothesised that the degree of leg activity would be an important determinant of the total daily activity of patients with COPD. In addition, leg activity would relate both to self-paced walking distance and muscle strength and would improve significantly after pulmonary rehabilitation. Moreover, we anticipated that the initial degree of activity impairment would predict the extent to which activity improved after rehabilitation, whether this was assessed objectively by activity monitoring or subjectively by activity questionnaire. To test these hypotheses, we have monitored activity simultaneously with a leg-mounted accelerometer and a Dynaport activity monitor and subsequently related leg activity to wellrecognised outcome measures before and after pulmonary rehabilitation.

\section{METHODS}

\section{Subjects}

Patients with a clinical and physiological diagnosis of $\mathrm{COPD}^{13}$ who had not used antibiotics or oral corticosteroids for at least 6 weeks and who were referred for pulmonary rehabilitation were recruited to the study. Medication was individually optimised before assessment and remained constant throughout the study. Patients using domiciliary oxygen, those with unstable cardiac disease, and those unable to exercise due to musculoskeletal, neurological or vascular disorders were excluded. All patients provided written informed consent and the protocol was approved by the local research ethics committee.

\section{Study protocol}

Patients participated in one or more of three study evaluations.

- Evaluation 1: A subset of patients with COPD underwent simultaneous leg accelerometry measurements and total body activity measurements. These subjects also completed health status and activity questionnaires.

- Evaluation 2: In a second group of patients with COPD, leg accelerometry measurements were compared with those of a control group of 
healthy volunteers of similar age and sex. Quadriceps muscle strength was also recorded.

- Evaluation 3: A third group of patients with COPD completed leg accelerometry measurements before and after pulmonary rehabilitation and these leg activity data were related to standard measures of lung function, exercise performance, muscle strength and health status.

Before rehabilitation, assessments were performed on two visits approximately 7 days apart. After rehabilitation, all assessments were completed on a single visit scheduled no more than 14 days after completion of the exercise programme. Each subject performed the same tests in the same order. All tests were performed before and after rehabilitation with the exception of lung function measurement and the two practice 6 min walks.

\section{Procedures}

\section{Pulmonary function tests}

Before testing, patients omitted short-acting inhaled bronchodilators for $8 \mathrm{~h}$ and long-acting $\beta$ agonists for $12 \mathrm{~h}$. Spirometry, static lung volumes and single breath carbon monoxide transfer factor were measured with a rolling seal spirometer ( $\mathrm{P} \mathrm{K}$ Morgan, Kent, UK) according to American Thoracic Society guidelines. ${ }^{14}$ Static lung volumes were measured by helium dilution. Predicted values used were those of the European Coal and Steel Community. ${ }^{15}$

\section{Heath status and disability questionnaires}

Patients completed the St George's Respiratory Questionnaire $(\mathrm{SGRQ})^{16}$ and the Hospital Anxiety and Depression (HAD) questionnaire, ${ }^{17}$ while self-reported activity was assessed using the Nottingham Extended Activities of Daily Living (NEADL) questionnaire. $^{18}$

\section{Quadriceps muscle strength}

Maximum quadriceps strength was measured by isometric maximum voluntary contraction of the dominant quadriceps using a custom-built set up. Further details of this are included in the online supplement. Subjects performed three maximum voluntary contractions with a rest period of 1 min between efforts. Maximum quadriceps strength was defined as the peak value obtained from the three recordings.

\section{Six minute walking test}

The tests were performed in accordance with ATS recommendations ${ }^{19}$ with two additional practice walks at the initial assessment. Perceived breathlessness was scored immediately before exercise and at maximum exercise using the modified Borg scale. ${ }^{20}$

\section{Activity measurement}

\section{Leg accelerometry measurement}

Leg activity was measured using the Actiwatch Uniaxial Accelerometer (Cambridge Neurotechnology, Cambridge, UK). All recordings were made continuously over three weekdays with the exception of Evaluation 1 (comparison of whole body and lower limb activity) where only 2 days were recorded. Using a lightweight strap, the Actiwatch was positioned just above the dominant ankle and subjects only removed the device for bathing and then repositioned the Actiwatch immediately afterwards. The Actiwatch has an event marker button and subjects pressed this button on rising in the morning, going to bed at night and when the device was removed for bathing.
Subjects also documented when and why the activity monitor was removed. On the rare occasion a subject forgot to press the marker button, the written record or period of overnight inactivity was used to determine the actigram. Activity monitoring was performed before and after rehabilitation with the same Actiwatch.

The Actiwatch signal is measured 32 times per second and processed to provide the amount and duration of movement. This signal is expressed as an activity count which denotes the amplitude of the signal detected by the accelerometer. A vale of approximately 25 counts represents gravitational acceleration. Further details about the technical specifications are given in the online supplement. Data were expressed as an activity count, which is the sum of all the epochs within each $30 \mathrm{~s}$ period. Inactivity was expressed as an activity count of zero. Data extracted for analysis were aggregated over the three daytime periods and expressed as:

- Mean activity score: average value of each $30 \mathrm{~s}$ epoch throughout the waking day, including all periods of zero activity.

- Mean intensity of activity: average value of each 30 s epoch when activity was occurring throughout the waking day (excludes any period of zero activity).

- Percentage of time mobile: percentage of $30 \mathrm{~s}$ epochs throughout the waking day where an activity score of $\geqslant 1$ was recorded; any epoch with a mean activity score of 0 was labelled immobile, while any epoch with a score of $\geqslant 1$ was active, although a score of 1 represents a very low level of activity.

\section{Total body activity measurement}

The Dynaport Activity Monitor (McRoberts BV, Den Haag, Netherlands) is a lightweight device containing a triaxial accelerometer. It has previously been validated for use in patients with COPD. ${ }^{9}$ The device consists of a lightweight box enclosed in a neoprene belt worn anteriorly around the waist. The box is connected to a leg sensor which is worn around the upper third of the thigh. The signal recorded by the device precisely measures the time spent walking, cycling, standing, sitting or lying, and it also provides a measure of movement intensity during the recording period. The technical specifications of the activity monitor have been detailed previously. ${ }^{21}$

The Dynaport Activity Monitor provides a measure of overall activity (movement intensity) and a measure of intensity of activity (movement intensity during movement). In addition, it records the proportion of the day during which the subject was moving (time spent moving). Different activities can be classified and expressed as the proportion of the day spent walking, standing, sitting and lying down.

The Dynaport Activity Monitor was fitted and all subjects were provided with written instructions, spare batteries and an emergency contact number. The subjects were monitored for two consecutive days, a time period over which reliable results have been obtained previously. ${ }^{7}$ Monitoring lasted from rising in the morning until whatever time in the evening they had completed their usual daily activities.

\section{Pulmonary rehabilitation}

The 8-week outpatient pulmonary rehabilitation programme consisted of two supervised and one unsupervised 1-hour exercise session per week. Patients received an individualised regime of aerobic upper and lower limb exercises which included peripheral muscle strengthening and whole body endurance exercises. Further details of the programme are included in the 
Table 1 Baseline characteristics and number of subjects in each study evaluation

\begin{tabular}{|c|c|c|c|c|}
\hline & $\begin{array}{l}\text { Evaluation } 1 \text { (subjects } \\
\text { with COPD who had } \\
\text { concurrent DP and AW } \\
\text { measurements) }\end{array}$ & $\begin{array}{l}\text { Evaluation } 2 \text { (normal } \\
\text { subjects of similar age } \\
\text { who had AW } \\
\text { measurement) }\end{array}$ & $\begin{array}{l}\text { Evaluation } 3 \text { (subjects } \\
\text { with COPD who had } \\
\text { AW measurement } \\
\text { before and after PR) }\end{array}$ & $\begin{array}{l}\text { Evaluations } 2 \text { and } 3 \\
\text { (subjects with COPD } \\
\text { who had AW } \\
\text { measurement) }\end{array}$ \\
\hline No of subjects & 12 & 18 & 23 & 33 \\
\hline M:F & $9: 3$ & $8: 10$ & $12: 11$ & 17:16 \\
\hline Age (years) & $63(7)$ & $70(6)$ & $66(9)$ & $67(8)$ \\
\hline $\begin{array}{l}\text { Current:ex:never } \\
\text { smokers }\end{array}$ & $3: 9: 0$ & $5: 9: 4$ & $5: 18: 0$ & $7: 26: 0$ \\
\hline $\mathrm{FEV}_{1}(\mathrm{I})$ & $1.01(0.43)$ & $2.5(0.6)$ & $0.93(0.32)$ & $0.96(0.4)$ \\
\hline $\mathrm{FEV}_{1}(\%)$ & $33.4(12.2)$ & $105.1(17.7)$ & $36.4(11.6)$ & $38.2(12)$ \\
\hline FVC (I) & $2.83(0.93)$ & $3.3(0.8)$ & $2.27(0.46)$ & $2.36(0.9)$ \\
\hline $\mathrm{FEV}_{1} / \mathrm{FVC}$ & $0.36(0.07)$ & $0.77(0.04)$ & $0.41(0.13)$ & $0.42(0.12)$ \\
\hline IC $\%$ & $73.3(14.5)$ & NK & $71.6(15.8)$ & $77.6(20.8)$ \\
\hline Quadriceps MVC (N) & NK & $415(98)$ & $315(106)$ & $308(116)$ \\
\hline SGRO Total & $63.4(16.2)$ & NK & $62.1(13)$ & $62.2(12.5)$ \\
\hline SGRO Activity & $77.2(19.4)$ & NK & $79.1(13.1)$ & $80.1(13.7)$ \\
\hline NEADL & $18.9(2.3)$ & NK & $16.4(2.7)$ & $15.7(2.8)$ \\
\hline \multicolumn{5}{|l|}{ Actiwatch measures: } \\
\hline $\begin{array}{l}\text { Mean activity }\left(\times 10^{3}\right. \\
\text { counts/h) }\end{array}$ & $123(110)$ & $143(61)$ & $82(53)$ & $82(49)$ \\
\hline $\begin{array}{l}\text { Mean intensity of } \\
\text { activity }\left(\times 10^{3} \text { counts } / \mathrm{h}\right)\end{array}$ & $190(162)$ & $232(90)$ & $156(69)$ & $156(63)$ \\
\hline$\%$ of time mobile & $63.2(14.5)$ & $61.4(11.2)$ & 50 (13.9) & $50.8(15.4)$ \\
\hline
\end{tabular}

online supplement. Owing to the "rolling" nature of the programme, subjects who were unable to attend particular supervised session(s) were able to continue the programme beyond 8 weeks until they had attended 16 supervised sessions. Patients who attended 16 sessions were defined as "completers".

\section{Statistical analysis}

Group data are expressed as mean (SD) and subgroup data as mean (SE). Statistical analysis was performed using SPSS V.15.0 and Stats Direct 2.6 with significance set at $p<0.05$; $p$ values are recorded to three decimal places. Normal distribution was assessed using the Shapiro-Wilks test and all data were normally distributed except for the Actiwatch mean activity score and Actiwatch mean activity score when active. These data were

Table 2 Measurements of activity obtained by the Actiwatch (leg) and Dynaport (whole body) systems during the 2 days of recording in 12 patients with chronic obstructive pulmonary disease (Evaluation 1)

\begin{tabular}{lc}
\hline & Mean (SD) \\
\hline Actiwatch & $63.3(14.5)$ \\
Time spent mobile $(\%$ of recording) & $123(110)$ \\
Mean activity score $\left(\times 10^{3}\right.$ counts/h) & $190(162)$ \\
Mean intensity of activity $\left(\times 10^{3}\right.$ counts/h) & \\
& \\
Dynaport & $13.5(4.4)$ \\
Time spent moving (\%) & $0.2(0.1)$ \\
Movement intensity & $1.48(0.21)$ \\
Movement intensity during movement & $3.6(2.8)$ \\
Time spent walking (\%) & $32.1(15.2)$ \\
Time spent standing (\%) & $58.7(17.6)$ \\
Time spent sitting (\%) & $5.3(4.9)$ \\
Time spent lying down (\%)
\end{tabular}

logarithmically transformed to normalise the distribution. Paired and unpaired Student $t$ tests were used to detect differences in group data and Pearson's correlation coefficient was used to examine the association between individual parameters. Multiple linear regression analysis was performed with the primary explanatory variables being change in activity scores after pulmonary rehabilitation. A model was constructed to examine the following potential exploratory variables: forced expiratory volume in $1 \mathrm{~s}\left(\mathrm{FEV}_{1}\right)$ in litres, $\mathrm{FEV}_{1}$ percent predicted, ratio of $\mathrm{FEV}_{1}$ to forced vital capacity (FVC), carbon monoxide transfer factor (TLCO) percent predicted, quadriceps strength, 6 min walking distance, SGRQ score and NEADL score. The final model was constructed using a backwards stepwise procedure; at each step a variable was removed which reduced the amount of variation accounted for by the least amount. Using published data, we considered an improvement in 6 min walk distance of $54 \mathrm{~m}$ after completion of pulmonary rehabilitation to be clinically significant. ${ }^{3}$ From pilot data we established that a change in Actiwatch mean activity score of 30 corresponded with similar improvements in walking distance and an improvement in health status. Based on the change in these two measures, we established that 24 subjects had to complete evaluation 3 of the study to detect a difference in these outcomes with $80 \%$ power at a significance level of $5 \%$.

\section{RESULTS}

\section{Baseline characteristics}

The baseline characteristics of the patients with COPD and healthy volunteers are shown in table 1 .

\section{Evaluation 1: Relationship between leg and whole body activity in patients with COPD}

Fourteen subjects were studied and all completed 2 days of recording with the Actiwatch. However, Dynaport 

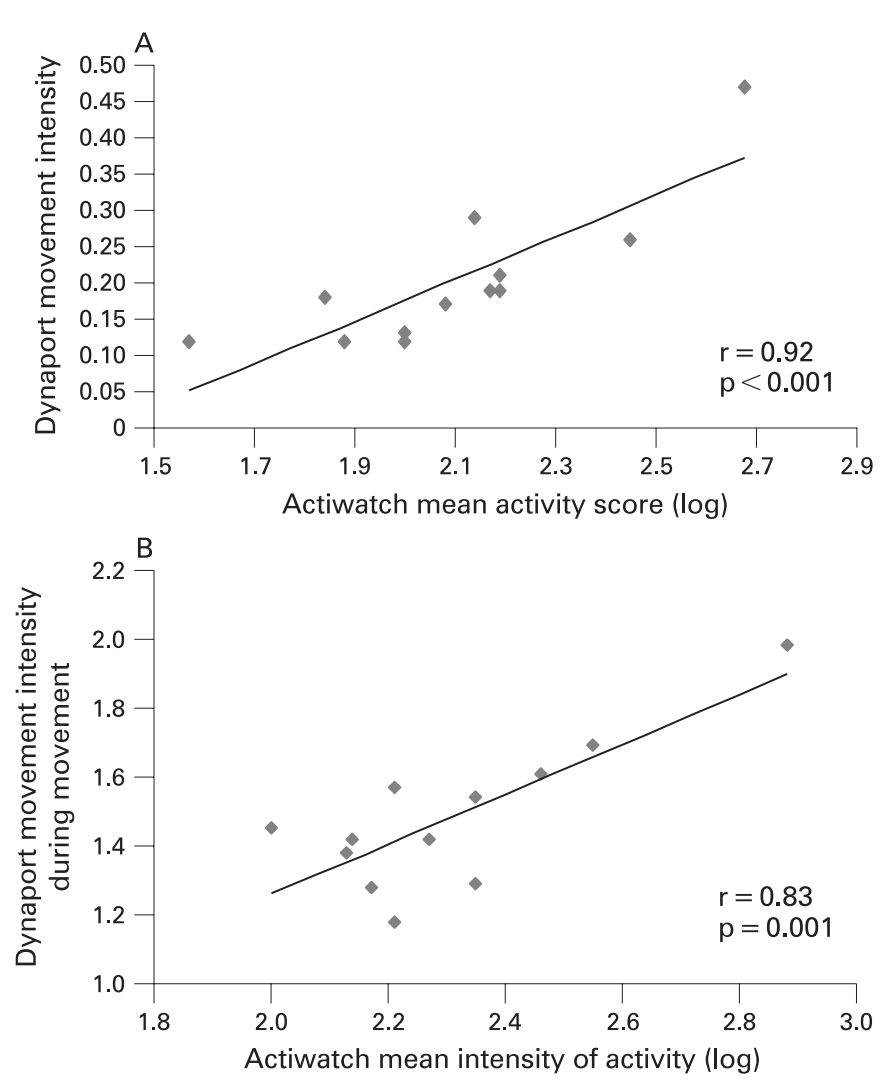

Figure 1 (A) Relationship between measures of mean activity by Dynaport and Actiwatch devices. (B) Relationship between measures of intensity of activity by Dynaport and Actiwatch devices.

measurements were unsuccessful in two patients owing to unrecognised lack of battery power (both patients) plus incorrect operation of the device by one patient. Simultaneously recorded data were therefore available in 12 patients with a mean recording duration of 18.7 (1.5) $\mathrm{h}$.

Mean data for the activity outcomes and body position are shown in table 2 . There was good agreement between the mean leg activity score recorded by the Actiwatch and the mean whole body activity level recorded by the Dynaport device (table 3 and fig 1A), and also between Actiwatch mean intensity of activity and all Dynaport activity assessments (table 3 and fig 1B). No relationship was seen between the time spent in specific positions and the activity levels assessed by the leg accelerometer. However, the mean number of counts recorded in each position for each patient was significantly different between sitting and walking (see table 1 in online data supplement).

\section{Evaluation 2: Leg activity in patients with COPD and healthy volunteers}

The patients and volunteers were of similar age and, by definition, differed in lung function (table 1). Quadriceps muscle strength was significantly less in the patients with COPD (difference $107 \mathrm{~N}, 95 \%$ CI 48 to 139; $p=0.001$ ). The patients spent significantly less of the day mobile (difference $10.6 \%, 95 \%$ CI $3.1 \%$ to $18.2 \%$; $p=0.007$ ) and had a lower mean activity level (difference 61, 95\% CI 27 to 96; $p=0.001$ ) and lower intensity of activity score (difference $77,95 \%$ CI 27 to $126 ; p=0.004)$ than the healthy volunteers. Leg activity recordings were relatively stable between the days for both the patients with COPD and the volunteers. The mean (range) coefficient of variation was $21.6 \%$ (2.2-47.4\%) for mean activity score, $15.7 \%(3.2-42 \%)$ for mean intensity of activity and $11.5 \%$ $(1.5-31.3 \%)$ for percentage of time spent mobile. When different leg activity measures recorded in the patients with COPD were compared, mean activity scores were closely related to the mean intensity of activity ( $r=0.86,95 \%$ CI 0.73 to $0.93 ; p<0.001)$ with a weaker relationship between the mean activity score and the percentage of time scored as mobile $(r=0.68,95 \%$ CI 0.44 to $0.83 ; \mathrm{p}<0.001)$. However, the intensity of activity when exercising was not related to the amount of time spent mobile $(\mathrm{r}=0.27,95 \% \mathrm{CI}-0.08$ to $0.56 ; \mathrm{p}=0.122)$.

\section{Evaluation 3a: Lower limb activity and laboratory assessments of exercise capacity}

In this evaluation the Actiwatch accelerometer was worn for an average of $15.7(0.2) \mathrm{h}$ per day on each of the 3 days of recording. All functional measurements-lung mechanics, muscle strength, walking distance or self-completed questionnaires - were related to each other to a varying degree (see table 2 in online data supplement). There was a significant or near significant relationship between measures of leg activity and many of these variables (see table 3 in online data

Table 3 Correlation between different activity measures obtained from the Actiwatch and Dynaport Activity Monitor in 12 patients with chronic obstructive pulmonary disease (Evaluation 1)

\begin{tabular}{llll}
\hline & Actiwatch & & \\
\cline { 2 - 4 } & Mean activity score & $\begin{array}{l}\text { Mean intensity of activity } \\
\text { score }\end{array}$ & Time spent mobile \\
\hline Dynaport & & \\
Time spent moving (\%) & $\mathrm{r}=0.83$ & $\mathrm{r}=0.76$ & $\mathrm{r}=0.48$ \\
& $95 \% \mathrm{Cl} 0.48$ to 0.95 & $95 \% \mathrm{Cl} 0.32$ to 0.93 & $95 \% \mathrm{Cl}-0.13$ to 0.32 \\
& $\mathrm{p}=0.001$ & $\mathrm{p}=0.004$ & $\mathrm{p}=0.118$ \\
Movement intensity & $\mathrm{r}=0.92$ & $\mathrm{r}=0.88$ & $\mathrm{r}=0.35$ \\
& $95 \% \mathrm{Cl} 0.72$ to 0.98 & $95 \% \mathrm{Cl} 0.63$ to 0.97 & $95 \% \mathrm{Cl}-0.28$ to 0.77 \\
Movement intensity during & $\mathrm{p}<0.001$ & $\mathrm{p}<0.001$ & $\mathrm{p}=0.27$ \\
movement & $\mathrm{r}=0.81$ & $\mathrm{r}=0.83$ & $\mathrm{r}=0.05$ \\
& $95 \% \mathrm{Cl} 0.45$ to 0.95 & $95 \% \mathrm{Cl} 0.49$ to 0.95 & $95 \% \mathrm{Cl}-0.54$ to 0.61 \\
Time spent walking (\%) & $\mathrm{p}=0.001$ & $\mathrm{p}=0.001$ & $\mathrm{p}=0.883$ \\
& $\mathrm{r}=-0.42$ & $\mathrm{r}=-0.32$ & $\mathrm{r}=-0.42$ \\
& $95 \% \mathrm{Cl}-0.8$ to 0.2 & $95 \% \mathrm{Cl}-0.76$ to 0.31 & $95 \% \mathrm{Cl}-0.8$ to 0.2 \\
\hline
\end{tabular}

Data recorded are Pearson's correlation coefficient, 95\% confidence interval of the correlation and $p$ value. 
Figure 2 Flow chart showing the outcome for the 33 subjects referred to the pulmonary rehabilitation (PR) programme.

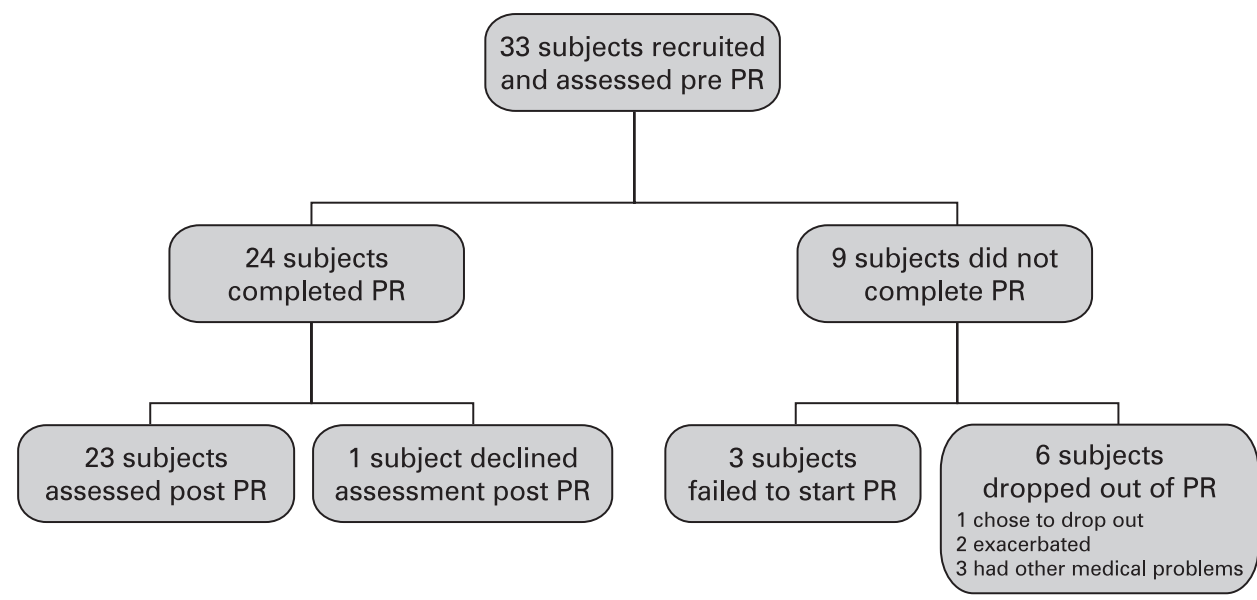

supplement). The mean activity of the legs and the percentage of time spent mobile were most closely related to absolute $\mathrm{FEV}_{1}$ ( $r=0.57,95 \%$ CI 0.28 to $0.76 ; \mathrm{p}<0.001$ and $\mathrm{r}=0.51,95 \% \mathrm{CI} 0.2$ to $0.73 ; p=0.002$, respectively). There was a significant relationship between $\mathrm{FEV}_{1}$ percent predicted and mean intensity of activity when exercising $(r=0.5,95 \%$ CI 0.19 to 0.72 ; $p=0.003)$, although in this case the best univariate correlation was with TLCO ( $r=0.6,95 \%$ CI 0.25 to $0.81 ; p=0.003)$. There was a weak relationship between mean leg activity and the subjective assessment of activity using the NEADL questionnaire $(r=0.35,95 \%$ CI 0 to $0.62 ; p=0.049)$.

\section{Evaluation 3b: Lower limb activity and pulmonary rehabilitation} The number of patients participating in and completing the pulmonary rehabilitation programme is shown in fig 2 . The change in outcome variables after completion of rehabilitation is presented in table 4. Subjects completing rehabilitation showed significant improvements in self-paced walking distance, quadriceps strength, breathlessness at rest and peak exercise, health status and level of anxiety and depression. In general, these changes exceeded the minimum clinically important difference and were paralleled by significant improvements in leg activity. However, the magnitude of change in walking distance and muscle strength were unrelated to the change in any index of leg activity.
Improvement in objectively measured leg activity was positively correlated with baseline $\mathrm{FEV}_{1}(\mathrm{r}=0.8,95 \%$ CI 0.58 to $0.91 ; p<0.001$, fig 3 ). The only other baseline variables that contributed to the improvement in any measure of activity were 6 min walk distance and NEADL score. Baseline subjective activity level assessed using the NEADL questionnaire was related to change in the percentage of time spent mobile $(\mathrm{r}=0.56,95 \%$ CI 0.15 to $0.79 ; \mathrm{p}=0.006)$ and mean activity score ( $r=0.47 ; 95 \%$ CI 0.08 to $0.71 ; p=0.021)$ after completion of rehabilitation. Full details of the relationships between change in leg activity and baseline variables are shown in table 4 in the online supplement.

\section{DISCUSSION}

Exercise limitation in COPD integrates the effect of many different aspects of this condition including abnormal lung mechanics, peripheral muscle dysfunction and altered mood states. Subjective assessments of exercise limitation range from simple reporting of activity limited by dyspnoea such as the MRC dyspnoea scale ${ }^{22}$ through to more global reporting of disability such as the SGRO activity score or NEADL. Cardiopulmonary exercise testing can define the physiological limits to exercise, but this and other objective exercise tests define what the patient can do rather than what they actually do at home. In comparison, recording accelerometry provides

Table 4 Effect of pulmonary rehabilitation in the 23 subjects with chronic obstructive pulmonary disease who completed all assessments (Evaluation 3 )

\begin{tabular}{|c|c|c|c|c|}
\hline & Before PR & After PR & $\begin{array}{l}\text { Mean (SE) difference } \\
\text { after PR }(95 \% \mathrm{CI})\end{array}$ & p Value \\
\hline Quadriceps MVC (N) & $312(20)$ & $334(23)$ & $22(31)(9$ to 35$)$ & 0.002 \\
\hline 6MWD (m) & $274(13)$ & $333(13)$ & 59 (33) (45 to 73) & $<0.001$ \\
\hline Resting Borg score & $1.6(0.2)$ & $0.9(0.2)$ & $0.7(1.2)(0.2$ to 1.6$)$ & 0.008 \\
\hline Peak Borg score & $4.6(0.2)$ & $3.2(0.3)$ & $1.4(1.7)(0.7$ to 2.1$)$ & $<0.001$ \\
\hline \multicolumn{5}{|l|}{ Leg activity } \\
\hline$\%$ of time spent mobile & $50.0(2.7)$ & $55.2(2.6)$ & $5.2(9.4)(1.2$ to 9.3$)$ & 0.014 \\
\hline Mean activity score $\left(\times 10^{3}\right.$ counts $\left./ \mathrm{h}\right)$ & $81.5(53.2)$ & $117.2(84.2)$ & $35.7(49)(14.5$ to 56.9$)$ & 0.002 \\
\hline Mean intensity of activity score $\left(\times 10^{3}\right.$ counts $\left./ \mathrm{h}\right)$ & $156(69.2)$ & $208.5(123.4)$ & $52.5(74.2)(20.4$ to 84.6$)$ & 0.001 \\
\hline \multicolumn{5}{|l|}{ Health status } \\
\hline HAD Anxiety & $7.2(1)$ & $5.6(0.6)$ & $1.6(3.2)(0.2$ to 3.0$)$ & 0.016 \\
\hline HAD Depression & $6.4(0.6)$ & $4.4(0.6)$ & $2(2.2)(1.0$ to 2.9$)$ & $<0.001$ \\
\hline SGRO & $62.9(2.5)$ & $47.8(2.4)$ & $15.1(12.5)$ (8.9 to 19.7$)$ & $<0.001$ \\
\hline NEADL & $16.4(0.5)$ & $18.2(0.5)$ & $1.8(1.7)$ (1.1 to 2.6$)$ & $<0.001$ \\
\hline
\end{tabular}

Data shown are mean (SE)

HAD, Hospital Anxiety and Depression questionnaire score; 6MWD, six minute walking distance (metres); MVC, maximum voluntary contraction; NEADL, Nottingham Extended Activities of Daily Living Questionnaire; PR, pulmonary rehabilitation; SGRO, St George's Respiratory Questionnaire. 

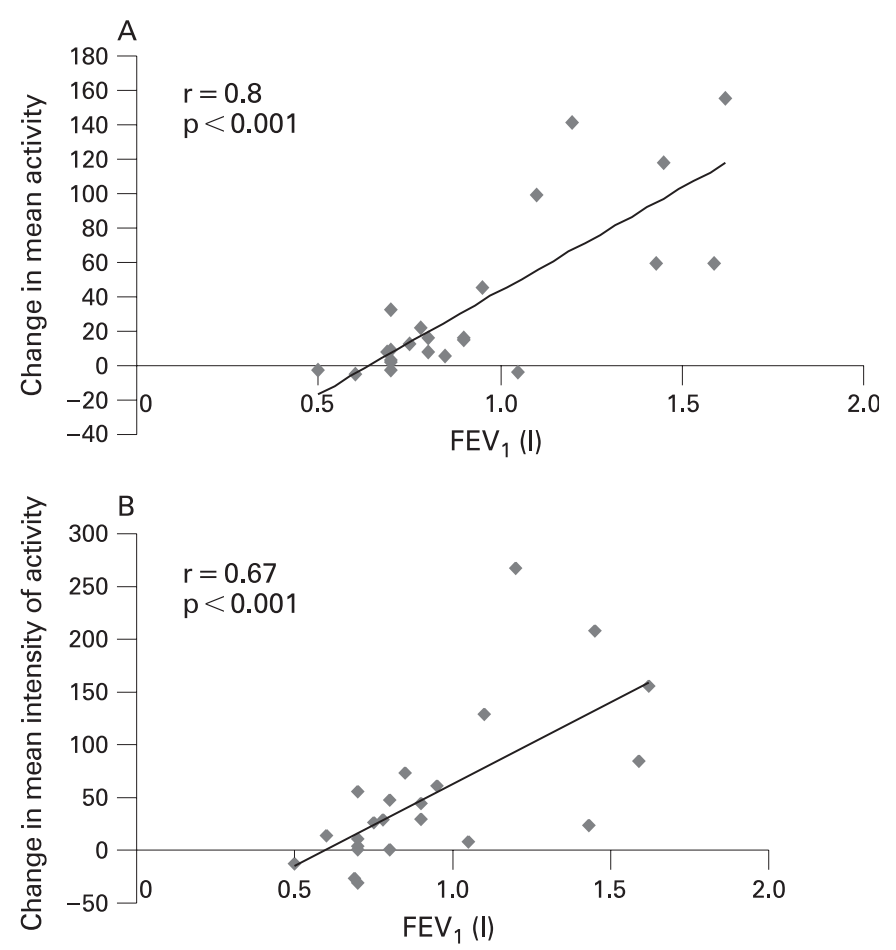

Figure 3 (A) Relationship between forced expiratory volume in $1 \mathrm{~s}$ $\left(\mathrm{FEV}_{1}\right)$ and change in Actiwatch leg mean activity after rehabilitation $(r=0.8, p<0.001)$. (B) Relationship between $\mathrm{FEV}_{1}$ and change in Actiwatch leg mean intensity of activity after rehabilitation $(r=0.67$, $\mathrm{p}<0.001$ ).

insight into how much activity is undertaken. In this report we have focused on leg activity and confirm that this variable relates well to whole body activity, distinguishes patients with COPD from unaffected individuals and responds to rehabilitation. However, interpreting these data is not necessarily simple, nor do they simply track changes in the variables we usually report when assessing a response to treatment.

Leg activity monitoring showed modest day-to-day variability in patients with COPD and healthy volunteers comparable to that reported with other systems. There was good agreement between the activity scores of the Dynaport, a validated whole body accelerometer, and both the mean activity scores and mean intensity of activity scores of the leg device. The differences in daily leg activity of the healthy volunteers and the patients with COPD are comparable to those reported for whole body activity, ${ }^{7}$ and improvements after rehabilitation suggest that leg actigraphy tracks other outcome measures and is responsive to intervention. The lack of agreement between leg activity and position monitoring data with the Dynaport system probably reflects the strict scoring standards we used to define mobility. However, we cannot exclude an effect due to leg movements when seated or lying. The lack of complete agreement between the two methods means that lower limb activity monitors cannot be substituted for those measuring total activity when determining whole body energy expenditure. ${ }^{23}$ Despite this, lower limb activity is clearly the major determinant of whole body activity and, in most circumstances, lower limb measurement is likely to be an acceptable surrogate for a whole body system.

Of the three leg accelerometric variables we reported, mean activity provided an acceptable compromise between both the percentage of time spent immobile and the mean intensity of activity during exercise. These latter variables were unrelated in the patients with COPD, which suggests that the amount of time spent sitting or lying completely still was determined by different factors from those determining intensity of lower limb activity when exercise had to be undertaken. This was reflected to a degree in the relative lack of relationship between lower limb activity and conventionally used performance indicators such as walking distance, quadriceps strength and activity questionnaires. Although these variables were related, the major factor determining leg activity was $\mathrm{FEV}_{1}$; patients with better lung function undertook a greater degree of activity. The relationship between lung function and walking distance was much weaker, albeit not dissimilar to published data, ${ }^{2}$ as was the relationship between activity and walking distance. Hence, level of activity does not appear simply to reflect capacity but may be affected by lifestyle and choice. The subjectively reported limitation in activity of daily living score was weakly related to mean level of activity, and similar associations were seen with other subjective scores. However, unlike objective activity measures, subjective scores did not relate to measures of pulmonary function. These data suggest that level and extent of activity at home are independent measures of function which can only be approximately assessed using currently available laboratory physiological outcomes or activity questionnaires.

Pulmonary rehabilitation produced significant improvements in health status, lower limb muscle strength and exercise performance that are comparable to the best results reported with other programmes. ${ }^{24}$ Significant improvement was seen in all measures of lower limb activity although, even after pulmonary rehabilitation, leg activity was still significantly less than that in age-matched controls. The magnitude of improvement in measures of functional capacity-such as walking distance and quadriceps strength-did not predict the change in leg activity at home, even though both were significantly greater after rehabilitation.

We initially hypothesised that the most inactive subjects would have a greater capacity to improve their level of activity after rehabilitation. In fact, the baseline level of activity was only weakly related to the improvement after rehabilitation, which suggests that even active individuals can further increase their level of activity with an effective rehabilitation programme. Instead, change in leg activity after rehabilitation, however expressed, was primarily influenced by lung function, although the pre-rehabilitation 6 min walking distance also made an independent contribution. Individuals with better exercise capacity and lung function thus did more at home and with greater intensity after completing rehabilitation. This is compatible with the subjective improvement in activity of daily living. As in previously published reports, ${ }^{25}$ patients with a better preserved $\mathrm{FEV}_{1}$ but worse perception of activity reported the greatest subjective benefit. The importance of spirometry as a predictor of outcome after rehabilitation is not entirely surprising as previous reports have highlighted the role of ventilatory capacity in determining improvement in walking distance after rehabilitation. ${ }^{26-28}$

The Actiwatch device was to be easy to use, acceptable to patients and had a low failure rate. As a simple strap-mounted device, it could be worn under clothing and, with a long battery life, it could be worn overnight and only needed to be removed for bathing. In contrast, the Dynaport was technically more difficult to use and, although light in weight, it was larger and noticeable. It includes both a waist and a leg strap and hence has to be removed overnight and during washing. The start-up procedure needs to be completed each morning and ideally both 
batteries and memory card should be changed daily. In choosing an activity monitor, the ease of use of the Actiwatch has to be balanced against the more precise body position activity data obtained with the Dynaport.

Our data provide further support for the usefulness of monitoring daily activity at home in patients with COPD and confirm that simple monitoring of leg movement gives useful insights into daily activity. The intensity and amount of leg activity a patient undertakes at home gives rather different results from those predicted by more conventional measurements such as walking distance, muscle strength and health status questionnaires. Understanding why people improve some forms of activity but not others after treatment and what determines how much of their improved exercise capacity they use after rehabilitation is an important area of future research which will be greatly aided by the availability of valid monitoring methods such as actigraphy.

Acknowledgements: The authors thank Mr Ashley Jones for his advice on the statistical analyses and Mrs Maureen Baldock for her help with typing

Funding: None.

Competing interests: None.

Ethics approval: All patients provided written informed consent and the protocol was approved by the local research ethics committee.

\section{REFERENCES}

1. Oga T, Nishimura K, Tsukino $M$, et al. Relationship between different indices of exercise capacity and clinical measures in patients with chronic obstructive pulmonary disease. Heart Lung 2002:31:374-81.

2. Pinto-Plata VM, Cote $\mathrm{C}, \mathrm{Cabral} \mathrm{H}$, et al. The 6-min walk distance: change over time and value as a predictor of survival in severe COPD. Eur Respir J 2004;23:28-33.

3. Redelmeier DA, Bayoumi AM, Goldstein RS, et al. Interpreting small differences in functional status: the six minute walk test in chronic lung disease patients. Am J Respir Crit Care Med 1997;155:1278-82.

4. Revill SM, Morgan MD, Singh SJ, et al. The endurance shuttle walk: a new field test for the assessment of endurance capacity in chronic obstructive pulmonary disease. Thorax 1999;54:213-22.

5. Garcia-Aymerich J, Farrero E, Felez MA, et al, Estudi del Factors de Risc d'Aguditzacio de la MPOC investigators. Risk factors of readmission to hospital for a COPD exacerbation: a prospective study. Thorax 2003:58:100-5

6. Garcia-Aymerich J, Lange $\mathrm{P}$, Benet $\mathrm{M}$, et al. Regular physical activity reduces hospital admission and mortality in chronic obstructive pulmonary disease: a population based cohort study. Thorax 2006;61:772-8.

7. Pitta F, Troosters T, Spruit MA, et al. Characteristics of physical activities in daily life in chronic obstructive pulmonary disease. Am J Respir Crit Care Med 2005; 171:972-7.
8. Sewell L, Singh SJ, Williams JE, et al. Can individualized rehabilitation improve functional independence in elderly patients with COPD? Chest 2005; 128:1194-200.

9. Pitta F, Troosters T, Spruit MA, et al. Activity monitoring for assessment of physical activities in daily life in patients with chronic obstructive pulmonary disease. Arch Phys Med Rehabil 2005;86:1979-85.

10. Bernard S, LeBlanc P, Whittom F, et al. Peripheral muscle weakness in patients with chronic obstructive pulmonary disease. Am J Respir Crit Care Med 1998;158:629-34.

11. Decramer $\mathbf{M}$, Gosselink $\mathrm{R}$, Troosters $\mathrm{T}$, et al. Muscle weakness is related to utilization of health care resources in COPD patients. Eur Respir J 1997;10:417-23.

12. Marquis K, Debigare $R$, Lacasse $Y$, et al. Midthigh muscle cross-sectional area is a better predictor of mortality than body mass index in patients with chronic obstructive pulmonary disease. Am J Respir Crit Care Med 2002;166:809-13.

13. Global Initiative for Chronic Lung Disease (GOLD). GOLD Workshop Report, 2006 update. www.goldcopd.com (accessed June 2007).

14. American Thoracic Society. ATS standardization of spirometry: 1994 update. Am J Respir Crit Care Med 1994:152:1107-36.

15. Quanjer $\mathbf{P H}$, Tammeling GJ, Cotes JE, et al. Lung volumes and forced ventilatory flows. Report of the Working Party on the Standardization of Lung Function Tests, European Community for Steel and Coal. Eur Respir J Supp/ 1993:16:5-40.

16. Jones PW, Quirk FH, Baveystock CM, et al. A self complete measure of health status for chronic airflow limitation. The St George's Respiratory Questionnaire. Am Rev Respir Dis 1992;145:1321-7.

17. Zigmond AS, Snaith RP. The hospital anxiety and depression scale. Acta Psychiatr Scand 1983;67:361-70.

18. Nouri FM, Lincoln NB. An extended activities of daily living scale for stroke patients Clin Rehabil 1987;1:301-5.

19. American Thoracic Society. ATS statement: guidelines for the six-minute walk test. Am J Respir Crit Care Med 2002;166:111-7.

20. Borg GAV. Psychophysical basis of perceived exertion. Med Sci Sports Exerc 1982;14:377-87.

21. Brandes $\mathbf{M}$, Rosenbaum D. Correlations between the step activity monitor and the DynaPort ADL-monitor. Clin Biomech 2004;19:91-4.

22. Bestall JC, Paul EA, Garrod R, et al. Usefulness of the Medical Research Council (MRC) dyspnoea scale as a measure of disability in patients with chronic obstructive pulmonary disease. Thorax 1999;54:581-6.

23. Bouten CV, Verboeket-van de Venne WP, Westerterp KR, et al. Daily physical activity assessment: comparison between movement registration and doubly labelled water. J Appl Physiol 1996;81:1019-26.

24. Troosters $\mathbf{T}$, Casaburi $\mathrm{R}$, Gosselink $\mathrm{R}$, et al. Pulmonary rehabilitation in chronic obstructive pulmonary disease. Am J Respir Crit Care Med 2005;172:19-38.

25. Okubadejo AA, O'Shea L, Jones PW, et al. Home assessment of activities of daily living in patients with severe chronic obstructive pulmonary disease on long-term oxygen therapy. Eur Respir J 1997;10:1572-5.

26. Zu Wallack RL, Patel K, Reardon JZ, et al. Predictors of improvement in the 12minute walking distance following a six-week outpatient pulmonary rehabilitation program. Chest 1991;99:805-80.

27. Troosters T, Gosselink R, Decramer M. Exercise training in COPD: how to distinguish responders from nonresponders. J Cardiopulm Rehabil 2001;21:10-7.

28. Plankeel JF, McMullen B, Macintyre NR. Exercise outcomes after pulmonary rehabilitation depend on the initial mechanism of exercise limitation among nonoxygen-dependent COPD patients. Chest 2005;127:110-6.

Stay a step ahead with Online First

We publish all our original articles online before they appear in a print issue. This means that the latest clinical research papers go straight from acceptance to your browser, keeping you at the cutting edge of medicine. We update the site weekly so that it remains as topical as possible. Follow the Online First link on the home page and read the latest research. 